\title{
EXTENSION HYBRID FORCE-POSITION ROBOT CONTROL IN HIGHER DIMENSIONS
}

\author{
VICTOR VLADAREANU, FLORENTIN SMARANDACHE, LUIGE VLADAREANU
}

\begin{abstract}
The paper presents an advanced method for solving contradictory problems of hybrid position-force control of the movement of walking robots by applying a 2D Extension Set. Using the linear and non-linear attraction point principle and the network of attraction curves, there is determined the 2D space Dependent Function generated by position and force in order to solve the robot real time control. The generalization of the extension distance and dependent function uses Extenics in Higher Dimensions theory eliminates the crisp logic matrix of Cantor logic which describes the position-force sequences. Thus was developed an optimization method for hybrid position-force control which ensures positioning precision and robot movement stability on rough terrain. The final conclusions lead to development of a methodology that allows obtaining high level results for hybrid position-force control using extended transformations onto the real numbers set and an optimization function generated by the extended dependence function in $2 \mathrm{D}$ space.
\end{abstract}

Keywords: extenics in higher dimensions, hybrid force-position control, linear and non-linear attraction point principle, the network of attraction curves

\section{Introduction}

A safe and robust behaviour of robots and mechatronic systems in contact with objects in their environment is the basic requirement for accomplishing tasks according to the given application. Stable control of the object - robot interaction implies a difficult technical problem. Thus, for contact control a simple method called ,position adaptation” is proposed by Whitney (1977) in which the contact force is used to modify the trajectory of the reference position of the robot's endeffector. Control of the arching movement, which in essence is force control implicitly based on position, was suggested by Lawrence and Stoughton (1987) and Kazerooni, Waibel and Kim (1990). Salisbury (1980) presented an active control method of apparent rigidity of the robot endeffector in Cartesian space. In this method the reference position used as input to control the contact force and no reference point for force are used. Raibert, Craig (1981) and Manson (1980) ensure force and position control when the robot interacts with the environment by decomposing it into „position sub-space” and „force sub-space”. These two sub-spaces correspond to the robot's movement directions, respectively free movement or constrained environment.

There is a growing interest to this problem, based on the research done by Pelletier and Daneshmend (1990), Lacky and Hsia (1991) and Chan (1991). Pelletier and Daneshmend present a blueprint for the adaptive control device which would be used to compensate for the variations of the environment rigidity during movement. However, they then discovered this is subject to instabilities. Lasky and Hsia describe a control device system consisting of a conventional impedance control device in the inner loop and a trajectory alteration control device in the outer loop for tracking the force, but their proposal is based on the science and calculation of the manipulative dynamic model. Chan develops a control device with variable structure for impedance control under parameter uncertainty and outside distrubance, but this strategy requires exact knowledge of the location and rigidity of the environment in order to obtain good force control. 
Extenics was developed by Cai Wen in 1983 and developed succesively, with a major impact in the scientific world of the last few years through results in e-learnig, data mining, image recognition, robotics, statistics and management research, among others [1, 2, 7, 10]. Extension set theory is a mathematical form for representing uncertainty which is an extension of classic set theory, with applications in many research fields [12-14]. Extenics is a field of study which aims to solve contradictory problems, as is the case of position - force control in the field of robotics, mechatronics and real time control.

\section{Architecure of the Explicit Position-Force Control System.}

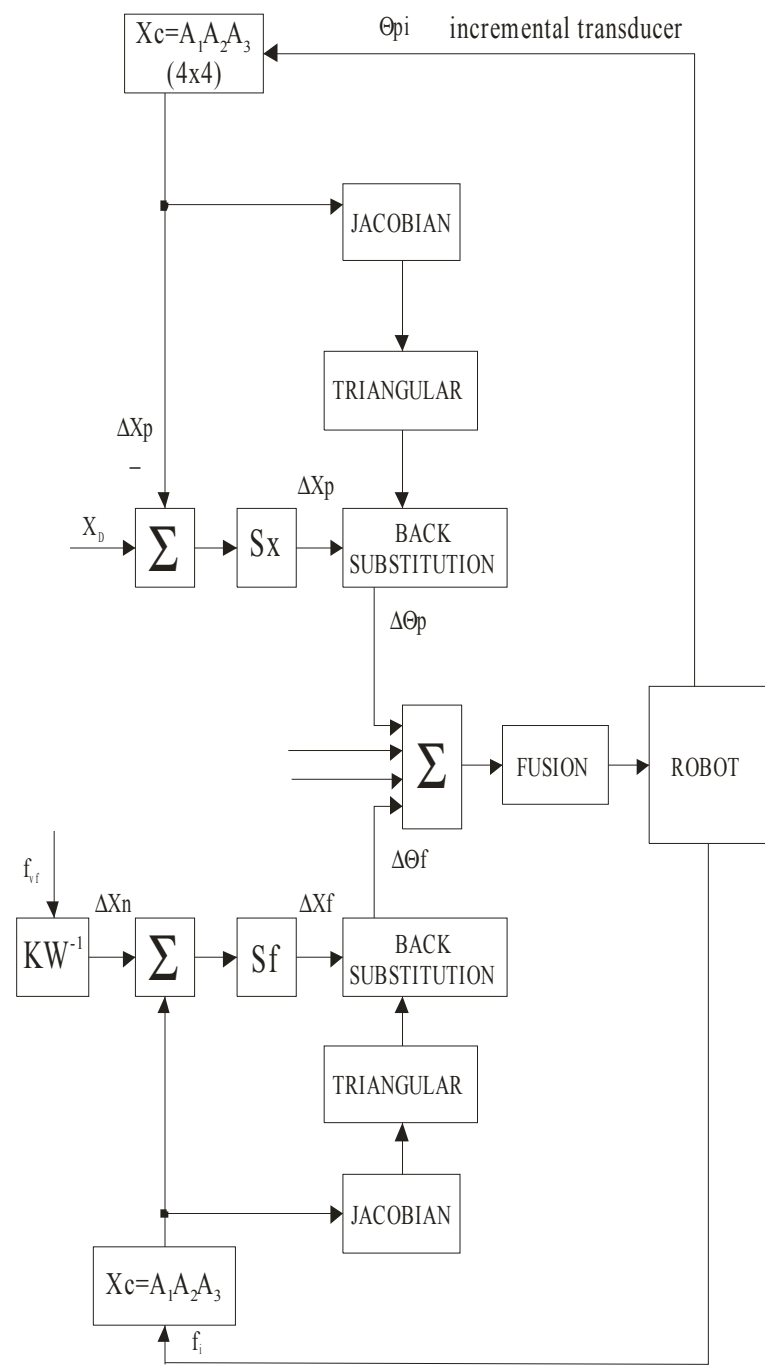

Fig.1. Architecure of the Position-Force Control System.
A hybrid position-force control system normally achieves simultaneous control of position and force. In order to determine the control relations in this situation, one divides the $\Delta \mathrm{X}_{\mathrm{P}}$ deviation measured by the command system into Cartesian coordinates into two sets: $\Delta \mathrm{X}^{\mathrm{F}}$ - corresponding to the force controlled component and $\Delta \mathrm{X}^{\mathrm{P}}$ corresponding to position control with actuation on the axis, in accordance with the selection matrixes $S_{f}$ and $S_{x}[5,6]$. Through this approach certain Cartesian coordinates of the robot end-effector are controlled in position while others are controlled explicitly in force.

The separate processing using separate laws for position and force control requires significant preparation of the treatment of tasks and changes in the implementation of the control loops; additionaly, this method may generate instability problems, especially during the transition of free and constrained movement $[8,9]$.

There results the motion variation on the robot axis in relation to the end-effector motion variation from the relation: $\Delta \mathrm{q}=\mathrm{J}^{-1}(\mathrm{q}) \Delta \mathrm{X}_{\mathrm{F}}+\mathrm{J}^{-1} 1(\mathrm{q})$ $\Delta \mathrm{X}_{\mathrm{P}}$, where $\Delta \mathbf{X}_{\mathbf{F}}$ can be calculated from the relation: $\Delta \mathrm{X}_{\mathrm{F}}=\mathrm{K}_{\mathrm{F}}\left(\Delta \mathrm{X}^{\mathrm{F}}-\Delta \mathrm{X}_{\mathrm{D}}\right)$, and $\mathrm{K}_{\mathrm{F}}$ is the dimensional relation of the stiffness matrix. Noting $\mathbf{F}_{\mathbf{D}}$ as the desired residual force and $\mathbf{K}_{\mathbf{W}}$ the physical stiffness the following relation is obtained: $\Delta \mathrm{X}_{\mathrm{D}}=\mathrm{K}_{\mathrm{W}}^{-1} * \mathrm{~F}_{\mathrm{D}}$.

The architecture of the hybrid position - force control system of robots with six degrees of freedom based on the Denevit - Hartenberg transformations is presented in Figure 1. The device sensors are used in two ways. In position control, the information obtained from the sensors is used to compensate the deviation of the robots' joints, due to the load created by external forces, so that the apparent stiffness of the robot's joint system is emphasised [11]. In force control, the joint is used as a force sensor, so that the manipulator is led in the same direction as the force received from the sensors, allowing the desired contact force to be maintained.

\section{Extension Hybrid Force-Position Robot Control}

Extenics is the science that deals with solving contradictory problems. In this paper the aim is to solve the contradictory problem of hybrid position - force control of the movement of robotic and mechatronics systems by replacing the logic 0 and 1 values in the selection matrices Sx and Sf, 
pertaining to force - position sequences using Cantor logic, with values of the dependent function using extended distance. Moreover, through a domain extension transformation for position $\mathrm{S}_{\mathrm{Kx}}$, respectively a domain extension transformation for force $\mathrm{S}_{\mathrm{Kf}}$, a new selection matrix with correlation coefficients for position, respectively one force force, are obtained, which allows for the determination of the position error signals $\varepsilon_{\mathrm{Kx}}$ and force error signals $\varepsilon_{\mathrm{Kf}}$ with the aim of closing the control system loops in position and force.

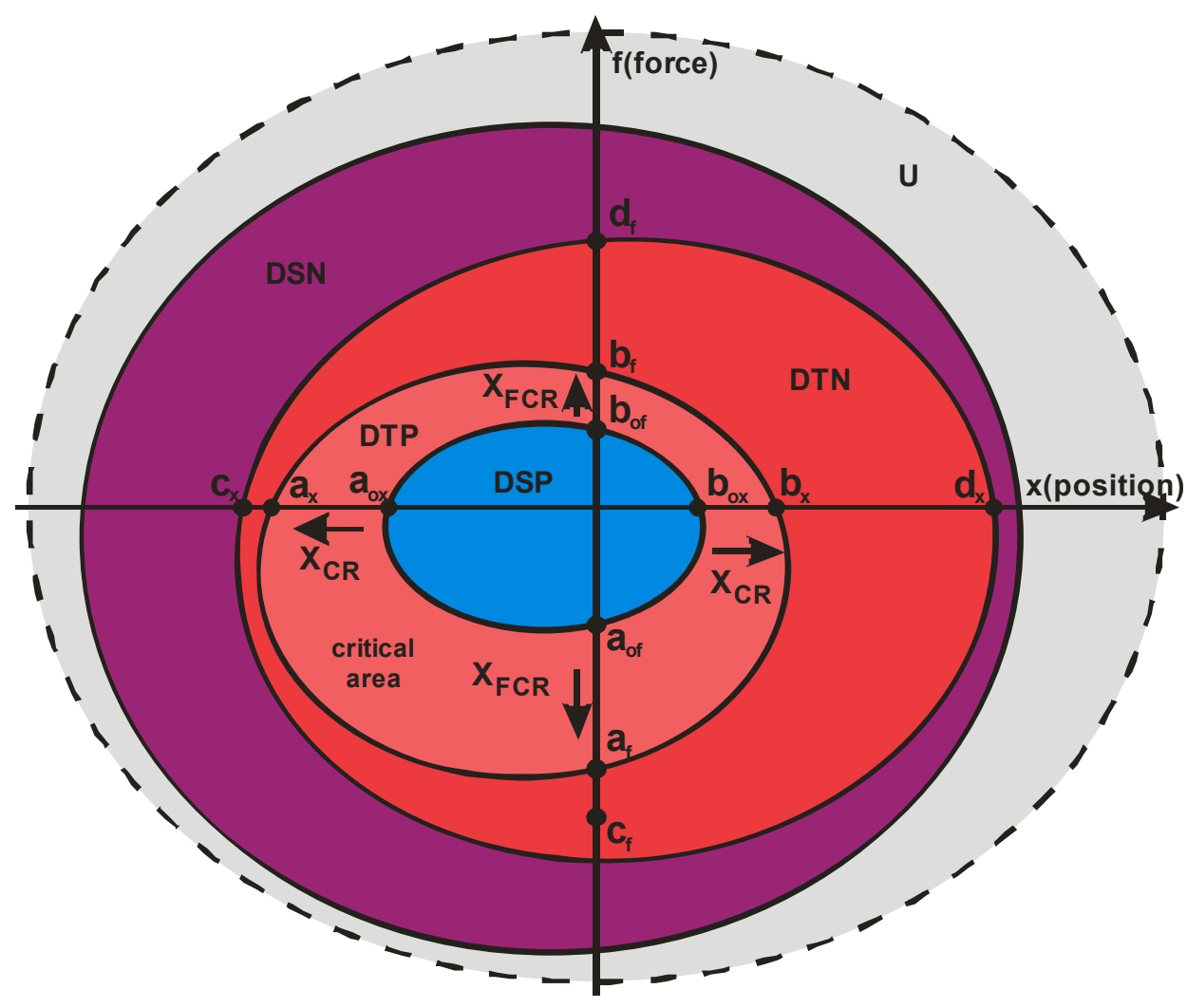

Fig. 2. Subdomains of the universe of discourse in reference to the position and force errors

In a first stage that is offline, the universe of discourse $U$ is defined for the position and force errors presented in Figure 2. Thus, the standard positive domain DSP is defined as the projection on the $\mathbf{x}$-axis of the acceptable position error for the reference $\mathbf{X}_{\mathbf{0}}$ of the movement of the robot system, nestled on the $\mathbf{x}$-axis in the interval $\left(a_{o x}, b_{o x}\right)$, where $a_{o x}$ and $b_{o x}$ are the negative and positive maximum acceptable position errors, respectively the projection on the $\mathbf{f}$-axis for the force reference of the movement $\mathbf{X}_{\mathbf{F o}}$, included in the interval $\left(a_{\text {of }}, b_{o f}\right)$, where $a_{\text {of }}$ and $b_{\text {of }}$ are the maximum accepted positive and negative force errors [15].

There results a positive transition domain DTP which projects onto the $\mathbf{x}$-axis as the positive transitive position interval $\mathbf{X}_{\mathbf{C R}}$ and onto the $\mathbf{f}$-axis as the positive transitive force interval $\mathbf{X}_{\mathbf{F C R}}$. The interval $\mathbf{X}_{\mathbf{C R}}$ corresponds to the critical error in position in which it is still possible to control the position movement of the robotic system in order to bring the position error into the standard positive domain. The $\mathbf{x}$-axis projection is limited by the interval $\left(a_{x}, b_{x}\right)$, where $a_{x}$ and $b_{x}$ are the positive, respectively negative maximum accepted critical errors. The interval $\mathbf{X}_{\mathbf{F C R}}$ corresponds to the critical error in force in which force control of the movement is still possible with the aim of bringing it into the standard positive domain and is limited by the interval $\left(a_{f}, b_{f}\right)$, where $a_{f}$ and $b_{f}$ are the positive, respectively negative maximum acceptable critical force errors.

A negative transition domain DTN which is continued with the standard negative domain DSN to compose the universe of discourse $U$ corresponds to unacceptable errors in position from which the position control of the robotic and mechatronic system SRM cannot recover to the standard positive domain, respectively unacceptable errors in force from which the force control cannot recover to the standard positive domain. Assigning values from this field would lead to saturation of the position or force reaction loop, with all negative consequences thereafter. The projection on the x-axis is 
nestled on the $\mathrm{x}$-axis in the interval $\left(\mathrm{c}_{\mathrm{x}}, \mathrm{d}_{\mathrm{x}}\right)$ where $\mathrm{c}_{\mathrm{x}}$ and $\mathrm{d}_{\mathrm{x}}$ are the negative and positive maximum unacceptable position errors, respectively the projection on the f-axis is included in the interval $\left(\mathrm{c}_{\mathrm{f}}\right.$, $d_{f}$ ) where $c_{f}$ and $d_{f}$ are the maximum unacceptable positive and negative force errors.

A standard negative domain DSN which completes the univers of discours $U$ and corresponds to unacceptable errors in position and force. The universe of discourse $U$ is composed of the sum of the domains presented before.

Having the defined domains of the universe of discourse, Linear and Non-Linear Attraction Point Principle is applied to the definition of metrics with the aim of determining the Linear (or Non-Linear) Dependent $n-D$-Function of point along the curve $c$ and the Extension $n$ - $D$-Distance between a point $P(x 1, x 2, \ldots, x n)$ and the $n-D$-set $\mathrm{S}$ as $\rho((x 1, x 2, \ldots, x n), \mathrm{S})$.

\section{Linear and Non-Linear Attraction Point Principle}

Optimal position-force control of a robot implies the tendency, in a linear or non-linear universe of discourse, towards an attraction point which ensures maximum performance.

The Linear and Non-Linear Attraction Point Principle is the following [3]:

Let $\mathrm{S}$ be an arbitrary set in the universe of discourse $\mathrm{U}$ of any dimension, and the optimal point $\mathrm{O} € \mathrm{~S}$. Then each point $P(x 1, x 2, \ldots, x n), n \geq 1$, from the universe of discourse (linearly or non-linearly) tends towards, or is attracted by, the optimal point $O$, because the optimal point $O$ is an ideal of each point. There could be one or more linearly or non-linearly trajectories (curves) that the same point $P$ may converge on towards $O$. Let's call all such points' trajectories as the Network of Attraction Curves (NAC).

It is a kind of convergence/attraction of each point towards the optimal point. There are classes of examples and applications where such attraction point principle may apply.

If this principle is good in all cases, then there is no need to take into consideration the center of symmetry of the set $\mathrm{S}$, since for example if one has a $2 D$ factory piece which has heterogeneous material density, then its center of weight (barycenter) is different from the center of symmetry.

Starting from the one-dimensional extension theory of Cai Wen, generalizations were necessary for $n$-dimensional spaces in order to define a measurable space and n-D extension distance with the aim of applying domain extension transformations in position $S_{\mathrm{Kx}}$ and force $\mathrm{S}_{\mathrm{Kf}}$ respectively, which would lead to solving the contradiction in robot hybrid force - position control.

1. We generalized in the track of Cai Wen's idea the extension $1 D$-set to an extension $n-D$ set, and defined the Linear (or Non-Linear) Extension $\boldsymbol{n}$ - $\boldsymbol{D}$-Distance between a point $P(x 1, x 2, \ldots$, $x n)$ and the $n-D$-set $\mathrm{S}$ as $\rho((x 1, x 2, \ldots, x n), \mathrm{S})$ on the linear (or non-linear) direction determined by the point $P$ and the optimal point $O$ (the line $P O$, or respectively the curvilinear $P O$ ) in the following way: the set $\mathrm{S}$;

a) $\rho((x 1, x 2, \ldots, x n), \mathrm{S})=$ the negative distance between $P$ and the set frontier, if $P$ is inside

b) $\rho((x 1, x 2, \ldots, x n), \mathrm{S})=0$, if $P$ lies on the frontier of the set $\mathrm{S}$;

c) $\rho((x 1, x 2, \ldots, x n), \mathrm{S})=$ the positive distance between $P$ and the set frontier, if $P$ is outside the set.

2. The Linear (or Non-Linear) Dependent $\boldsymbol{n}$ - $\boldsymbol{D}$-Function of point $P(x 1, x 2, \ldots, x n)$ along the curve $c$, is:

$$
K_{n D}\left(x_{1}, x_{2}, \ldots, x_{n}\right)=\frac{\rho_{c}\left(\left(x_{1}, x_{2}, \ldots, x_{n}\right), S_{2}\right)}{\rho_{c}\left(\left(x_{1}, x_{2}, \ldots, x_{n}\right), S_{2}\right)-\rho_{c}\left(\left(x_{1}, x_{2}, \ldots, x_{n}\right), S_{1}\right)}
$$

which has the following property:

a) If point $P(x 1, x 2, \ldots, x n) € \operatorname{Int}(\mathrm{S} 1)$, then $K n D(x 1, x 2, \ldots, x n)>1$;

b) If point $P(x 1, x 2, \ldots, x n) € \mathrm{Fr}(\mathrm{S} 1)$, then $K n D(x 1, x 2, \ldots, x n)=1$; 
c) If point $P(x 1, x 2, \ldots, x n) € \operatorname{Int}(\mathrm{S} 2-\mathrm{S} 1)$, then $K n D(x 1, x 2, \ldots, x n) €(0,1)$;

d) If point $P(x 1, x 2, \ldots, x n) € \operatorname{Int}(\mathrm{S} 2)$, then $K n D(x 1, x 2, \ldots, x n)=0$;

e) If point $P(x 1, x 2, \ldots, x n) € \operatorname{Int}(\mathrm{S} 2)$, then $K n D(x 1, x 2, \ldots, x n)<0$.

Let's see in figure 3 such example in the $2 D$-space.

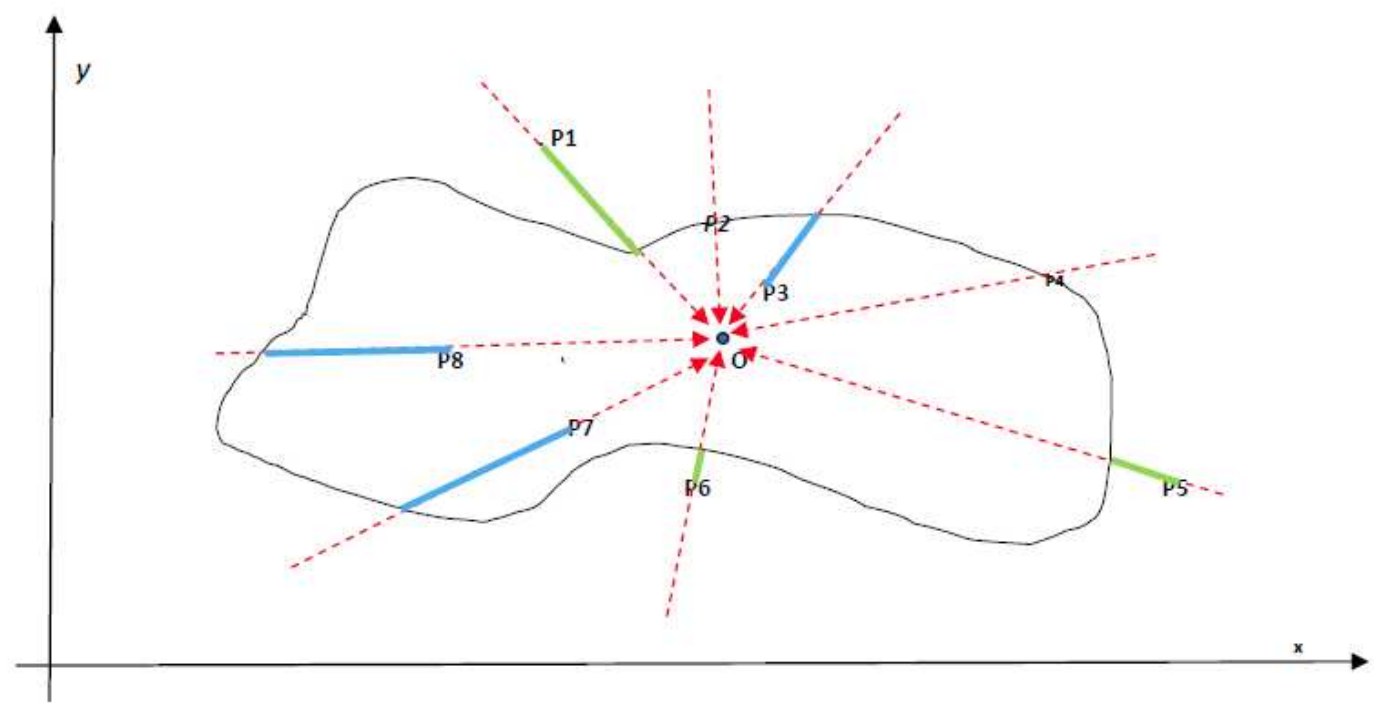

Fig. 3. The optimal point $\mathrm{O}$ as an attraction point for all other points $\mathrm{P} 1, \mathrm{P} 2, \ldots, \mathrm{P} 8$ in the universe of discourse R2

In general, in a universe of discourse $U$, let's have an $n-D$-set $\mathrm{S}$ and a point $P$.

Then the Extension Linear $\boldsymbol{n}$-D-Distance between point $P$ and set $\mathrm{S}$, is:

$$
\rho(P, S)=\left\{\begin{array}{cc}
-d\left(P, P^{\prime}\right), & P \neq O, P \in\left|O P^{\prime}\right| ; \\
P^{\prime} \in F r(S) & P \neq O, P^{\prime} \in|O P| ; \\
d\left(P, P^{\prime}\right), & P=O . \\
P^{\prime} \in \operatorname{Fr}(S) & \\
-\underset{M a x}{\max d(P, M)}, &
\end{array}\right.
$$

where $O$ is the optimal point (or linearly attraction point); $d\left(P, P^{\prime}\right)$ means the classical linearly $n-D$ distance between two points $P$ and $P^{\prime} ; F r(\mathrm{~S})$ means the frontier of set $\mathrm{S}$; and $\left|O P^{\prime}\right|$ means the line segment between the points $O$ and $P^{\prime}$ (the extremity points $O$ and $P^{\prime}$ included), therefore $P €\left|O P^{\prime}\right|$ means that $P$ lies on the line $O P^{\prime}$, in between the points $O$ and $P^{\prime}$.

For $P$ coinciding with $O$, one defined the distance between the optimal point $O$ and the set $\mathrm{S}$ as the negatively maximum distance (to be in concordance with the $1 D$-definition).

The Extension Non-Linear $\boldsymbol{n}$ - $\boldsymbol{D}$-Distance between point $P$ and set $\mathrm{S}$, is:

$$
\rho_{c}(P, S)=\left\{\begin{array}{cc}
-d_{c}\left(P, P^{\prime}\right), & P \neq O, P \in c\left(O P^{\prime}\right) ; \\
P^{\prime} \in F r(S) & P \neq O, P^{\prime} \in c(O P) ; \\
d_{c}\left(P, P^{\prime}\right), & P=O . F r(S) \\
-\operatorname{Pax}_{M \in F r(S), M \in c(O)} d_{c}(P, M), &
\end{array}\right.
$$


where $\rho c(P, S)$ means the extension distance as measured along the curve $c$; $O$ is the optimal point (or non-linearly attraction point); the points are attracting by the optimal point on trajectories described by an injective curve $c ; d c\left(P, P^{\prime}\right)$ means the non-linearly $n$ - $D$-distance between two points $P$ and $P^{\prime}$, or the arclength of the curve $c$ between the points $P$ and $P^{\prime}$;

$\operatorname{Fr}(\mathrm{S})$ means the frontier of set $\mathrm{S}$; and $\mathrm{c}\left(O P^{\prime}\right)$ means the curve segment between the points $O$ and $P^{\prime}$ (the extremity points $O$ and $P^{\prime}$ included), therefore $P \epsilon_{c}\left(O P^{\prime}\right)$ means that $P$ lies on the curve $c$ in between the points $O$ and $P^{\prime}$.

For $P$ coinciding with $O$, one defined the distance between the optimal point $O$ and the set $\mathrm{S}$ as the negatively maximum curvilinear distance (to be in concordance with the $1 D$-definition).

\section{The System Architecture for the Extension Hybrid Force-Position Robot Control.}

We intend to replace the $S_{x}$ and $S_{f}$ matrices which contain crisp logic values with $K_{x}$ and $K_{f}$ which contain values of the dependent function of the position error $K_{x}$ and the force error $K_{f}$ with respect to the standard positive field. Thus, considering the universe of discourse in figure 2 we will replace the crisp logic values 1 in matrices $S_{x}$ and $S_{f}$ with the $K_{x}$ and $K_{f}$ coefficients which result from the error positioning corresponding to the standard positive field [15].

The architecture of the Extension Position-Force Control System, presented in Figure 4, consists of a series of modules whose aim is to solve the contradictory problem of hybrid position force control for the movement of robotic and mechatronic systems. This is obtained conceptually by replacing the 0 and 1 logic values from the selection matrices Sx and Sf, depending on the position-force sequences in Cantor logic, with values of the dependent function using extension distance. This is followed by a domain extension transformation for position $\mathrm{S}_{\mathrm{Kx}}$, respectively for force $\mathrm{S}_{\mathrm{Kf}}$, which generates a new selection matrix with correlation coefficients for position and force respectively.

Thus, a module which calculates the position extension distance CDEP, receives the current position signal $\mathrm{X}$ processed by a Carthesian coordinate calculation module CCC through direct cinematic, of the robotic and mechatronic system SRM and in reference to the standard positive interval of the position reference $\mathrm{X}_{0}$, defined experimentally, calculates the position extension distance $\rho\left(X, X_{0}\right)$, which it sends to the module calculating the position dependence function CFDP. The extension position distance $\rho\left(X, X_{0}\right)$, according to extension theory, is calculated as the distance from a point, in this case the current position signal $\mathrm{X}$, to an interval, in this case the standard positive interval for reference position $\mathrm{X}_{\mathrm{o}}$. Similarly, the data for calculating the force extension distance is calculated by the CDEF module, which works quasi-simultaneously with the CDEP module which calculates the extension position distance.

The position dependent function $\mathrm{K}\left(\mathrm{X}, \mathrm{X}_{\mathrm{o}}, \mathrm{X}_{\mathrm{CR}}\right)$ of the current position signal $\mathrm{X}$ in relation to the standard positive interval of the reference position $\mathrm{X}_{\mathrm{o}}$ and the transitive positive interval for position $\mathbf{X}_{\mathbf{C R}}$ is determined according to extension theory in the CFDP module. This has the maximum value of $\mathrm{K}\left(\mathrm{X}_{\mathrm{o}}\right)=\mathrm{M}_{\mathrm{P}}$ equal to the proportional amplification component of the position controller on the standard positive interval for the reference position $\mathrm{X}_{\mathrm{o}}$. Moreover, in order to not saturate the position loop, the dependent function for position $\mathrm{K}\left(\mathrm{X}, \mathrm{X}_{\mathrm{o}}, \mathrm{X}_{\mathrm{CR}}\right)$ has a lower limit of 0 if the current position signal $\mathrm{X}$ is within the intervals $\mathrm{X}_{\mathrm{o}}$ and $\mathbf{X}_{\mathbf{C R}}$. 


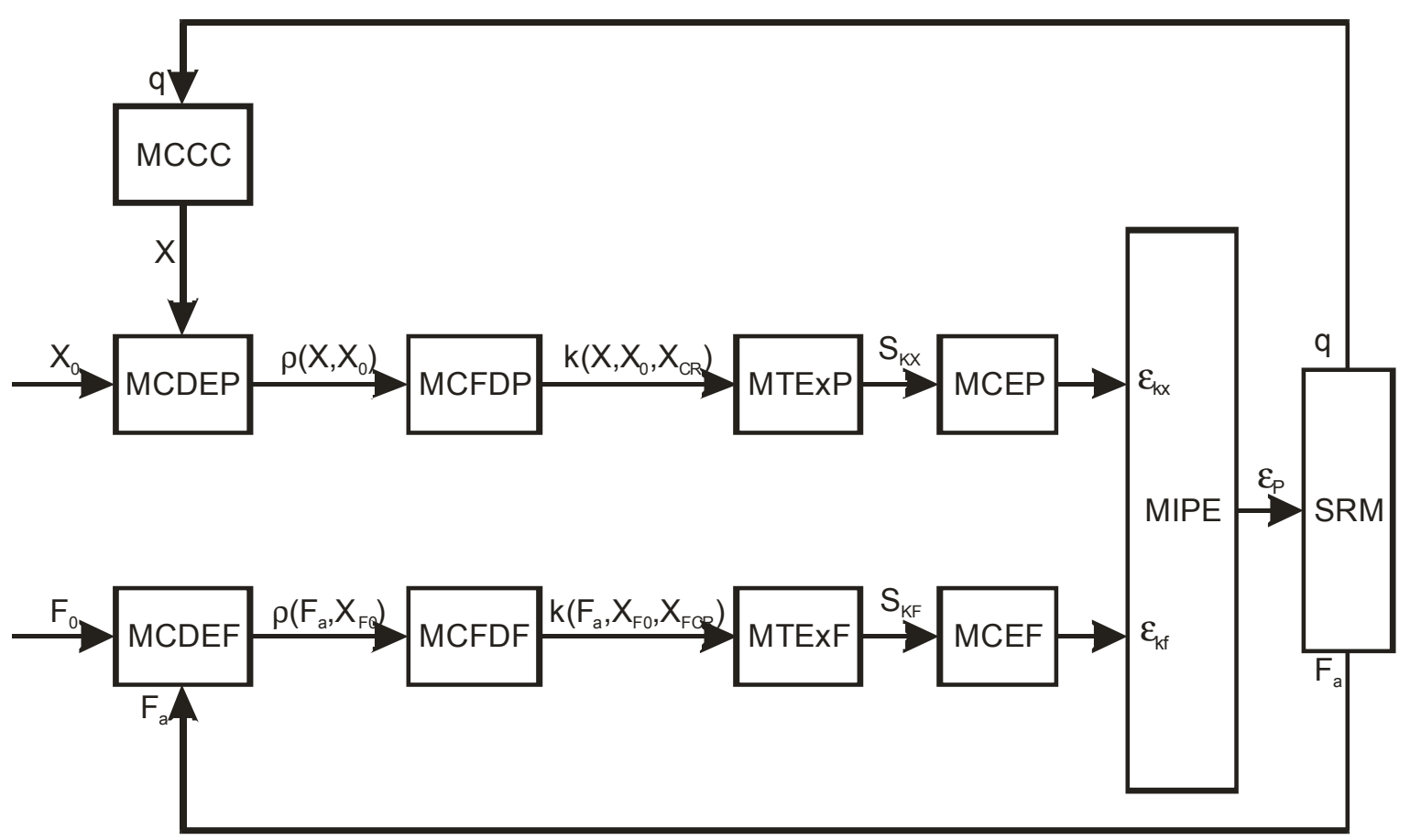

Fig. 4. Architecture of the extension hybrid force-position control system

Dependent function $\mathrm{k}(\mathrm{x}), \mathrm{k}(\mathrm{f})$ to enable the calculation of correlation do not have to rely on subjective judgments or statistics, it can quantitatively and objectively describe the elements having the nature or character at a certain extent and the process of quantitative change and qualitative change. This allows correlation function out of bias caused by subjective judgments.

Appling equations (1)-(3) for 2D, in a universe of discourse $U$, let's have a nest of two $n-D$ sets, S1 C S2, with no common end points, and a point $P$.

Then the Extension Linear Dependent $\boldsymbol{n}$-D-Function referring to the point $P(x 1, x 2, \ldots$, $x n)$ is:

$$
K_{n D}(P)=\frac{\rho\left(P, S_{2}\right)}{\rho\left(P, S_{2}\right)-\rho\left(P, S_{1}\right)}
$$

where $\rho(P, S 2)$ is the previous extension linear n-D-distance between the point $P$ and the $n$ - $D$-set S2.

The Extension Non-Linear Dependent n-D-Function referring to point $P(x 1, x 2, \ldots, x n)$ along the curve $c$ is:

$$
K_{n D}(P)=\frac{\rho_{c}\left(P, S_{2}\right)}{\rho_{c}\left(P, S_{2}\right)-\rho_{c}\left(P, S_{1}\right)}
$$

where $\rho c(P, S 2)$ is the previous extension non-linear n-D-distance between the point $P$ and the $n-D$ set $\mathrm{S} 2$ along the curve $c$.

Applications of the Extenics force-position control to 2D-Space in figure 5 is presented. We have a errors domain whose desired $2 D$-dimensions should be $20 \mathrm{mV}$ x $30 \mathrm{mV}$, and acceptable $2 D$ dimensions $22 \mathrm{mV} \times 34 \mathrm{mV}$. We define the extension 2D-distance, and then we compute the extension 2D-dependent function. 


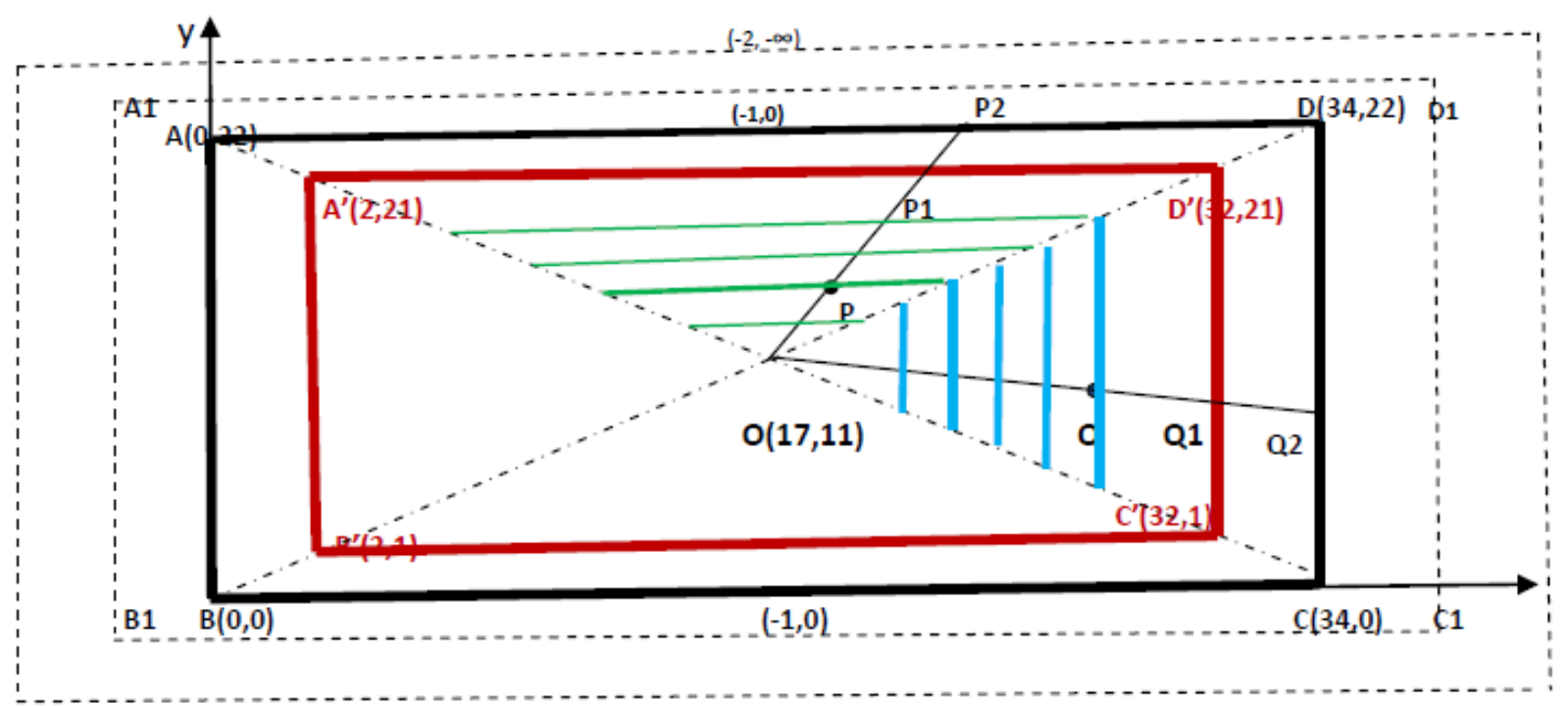

Fig. 5. Diagram of the extension 2D-dependent function

We have a desirable domain A'B'C'D' and an acceptable domanin ABCD [4]. The optimal opoint for both of them is $\mathrm{O}(17,11)$.

a) The region determined by the rays OA and OD.

The extension 2D-distance $\rho$ between a point $\mathrm{P}$ and a set is the \pm distance from $\mathrm{P}$ to the closestfrontier of the set, distance measured on the line OP. Whence

$$
\begin{aligned}
& \rho\left(\mathrm{P}, \mathrm{A}^{\prime} \mathrm{B}^{\prime} \mathrm{C}^{\prime} \mathrm{D}^{\prime}\right)=-|\mathrm{PP} 1| \\
& \rho(\mathrm{P}, \mathrm{ABCD})=-|\mathrm{PP} 2| .
\end{aligned}
$$

The extension 2D-dependent function $k$ of a point $\mathrm{P}$ which represents the dependent of the point of the nest of the two sets is:

$$
k(P)= \pm \frac{\rho(P, \text { bigger_set })}{\rho(P, \text { bigger_set })-\rho(P, \text { smaller_set })}= \pm \frac{\rho(P, A B C D)}{\rho(P, A B C D)-\rho\left(P, A^{\prime} B^{\prime} C^{\prime}\right)}= \pm \frac{\left|P P_{2}\right|}{\left|P P_{2}\right|-\left|P P_{1}\right|}= \pm \frac{\left|P P_{2}\right|}{\left|P_{1} P_{2}\right|} .
$$

In other words, the extension 2D-dependent function $\mathrm{k}$ of a point $\mathrm{P}$ is the $2 \mathrm{D}$-extension distance between the point and the closest frontier of the larger set, divided by the 2D-extension distance between the frontiers of the two nested sets; all these 2D-extension distances are taken along the line OP.

b) The region determined by the rays $O C$ and $O D$. Similar result would obtain if one gets the opposite region determined by the rays $O A$ and $O B$. If one takes another region determined by the rays $\mathrm{OC}$ and $\mathrm{OD}$ and a point $\mathrm{Q}(\mathrm{x} 1, \mathrm{y} 1)$ in between one gets:

$$
k(Q)=k\left(x_{1}, y_{1}\right)= \pm \frac{\left|Q Q_{2}\right|}{\left|Q_{1} Q_{2}\right|}
$$

A complete calculation is presented in [4].

A new selection matrix $\mathrm{S}_{\mathrm{Kx}}$ with the correlation coefficients for position is generated by the extension transformation module for position TExP which receives the dependent function signal for position $\mathrm{K}\left(\mathrm{X}, \mathrm{X}_{0}, \mathrm{X}_{\mathrm{CR}}\right)$ from the CFDP module and replaces the elements with value 1 in the position selection matrix with correlation coefficients for position, determined through an extension transformation in domain. For force similar processing takes place by using the CDEF, CFDF, TExF AND CEF modules, while quasi-simultaneously applying the position processing.

By applying the explicit sequential force-position control method on the two components, position in the CEP module and force in the CEF module, the position error and force error signals $\varepsilon_{\mathrm{Kx}}$ and $\varepsilon_{\mathrm{Kf}}$ respectively are determined with the aim of closing the system control loop in position and in force. 
The implementation methodology of this advance method for hybrid position-force control of the walking robot consists in determining experimentally the standard positive field and the transient positive field for each control component, applying the transformation on the force and position error taking into account their real position in relation to the standard positive field, defined by points $\mathrm{a}_{0 \mathrm{x}}$ and $\mathrm{b}_{0 \mathrm{x}}$ for position, respectively $\mathrm{a}_{0 \mathrm{f}}$ and $\mathrm{b}_{0 \mathrm{f}}$ for force, resulting in a transformed position and force error which represents the optimized function for hybrid position-force control.

The universe of discourse is configured to admit a transient negative field, defined by points $c_{x}$ and $d_{x}$ for position, respectively $c_{f}$ and $d_{f}$ for force, so that passing these points the position and force errors will be limited so as not to lead to controller saturation and all the negative effects that derive from it.

\section{Results and conclusions}

The obtained results lead to an advanced method of solving the contradictory problem of hybrid position-force control for robot movement by applying an "Extension Set", which allows the two contradictory elements, force and position, to be controlled simultaneously in real time, allowing for improvements in the movement precision and stability of the robot. Starting from the extended distance given by Prof. Cai Wen the dependent function in 2D space generated in position and force is determined.

By replacing crisp logic values in the $S_{x}$ and $S_{f}$ matrices depending on the force-position sequence with values of the Extension Distance and Dependent Function for 2D space given by Smarandache, a method is developed for optimizing hybrid position-force control which ensures positioning precision and stability for the robot.

The final conclusion lead to the development of a methodology which will allow high level results for hybrid force-position control, by using an extended transformation using as an optimization function the dependence function based on extension distance, in comparison to the classical method using sequential matrices corresponding to Cantor logic.

\section{References:}

[1] Cai Wen. Extension Set and Non-Compatible Problems [J]. Journal of Scientific Exploration, 1983, (1): 83-97.

[2] Yang Chunyan, Cai Wen. Extension Engineering [M]. Beijing: Science Press, 2007.

[3] F Florentin Smarandache, "Extenics in Higher Dimensions", Education Publisher, USA, pp.114, cap.1, "Generalizations of the Distance and Dependent Function in Extenics to 2D, 3D, and nD", pg. 1-21, ISBN: 9781599732039

[4] Smarandache Florentin, Vladareanu Victor, Applications of Extenics to 2D-Space and 3DSpace, The 6th Conference on Software, Knowledge, Information Management and Applications, Chengdu, China.Sept. 9-11, 2012 pp.12

[5] Luige Vladareanu1, Gabriela Tont, Ion Ion, Victor Vladareanu, Daniel Mitroi, Modeling and Hybrid Position-Force Control of Walking Modular Robots, ISI Proceedings, Recent Advances in Applied Mathematics, Harvard University, Cambridge, USA, 2010, pg. 510-518, ISBN 978 960-474-150-2, ISSN 1790-2769.

[6] Vladareanu L., Capitanu L., „Hybrid Force-Position Systems with Vibration Control for Improvment of Hip Implant Stability” Journal of Biomechanics, vol. 45, S279, Elsevier, 2012.

[7] Cai Wen. Extension Theory and Its Application [J]. Chinese Science Bulletin, 1999, 44(17): $1538-1548$

[8] An C.H., Hollerbach J.M., The Role of Dynamic Models in Cartesian Force Control of Manipulators, The International Journal of Robotics Research,Vol.8,No.4, August 1989,pg. 5171 
[9] Fisher W.D., Mujtaba M.S. - Hybrid Position/Force Control: A Correct Formulation, The International Journal of Robotics Research, Vol. 11, No. 4, August 1992, pp. 299-311.

[10] Yang Chunyan. The Methodology of Extenics [A]. Extenics: Its Significance in Science and Prospects in Application[C]. The 271th Symposium's Proceedings of Xiangshan Science Conference, 2005, 12: 35-38.

[11] Vladareanu, L., Tont, G., Ion, I., Munteanu, M. S., Mitroi, D., "Walking Robots Dynamic Control Systems on an Uneven Terrain", Advances in Electrical and Computer Engineering, ISSN 1582-7445, e-ISSN 1844-7600, vol. 10, no. 2, pp. 146-153, 2010, doi: 10.4316/AECE.2010.02026

[12] Guan Feng-Xu, Wang Ke-Jun. Study on Extension Control Strategy of Pendulum System [J]. Journal Of Harbin Institute Of Technology, 2006, 38(7): 1146-1149.

[13] Wong Chingchang, Chen Jenyang. Adaptive Extension Controller Design for Nonlinear Systems [J]. Engineering Science, 2001, 3 (7): 54-58.

[14] Zhi Chen, Yongquan Yu. To Find the Key Matter-Element Research of Extension Detecting [A]. Int. Conf. Computer, Communication and Control Technologies(CCCT) [C]. Florida, USA, $2003,7$.

[15] L. Vladareanu, Cai Wen, R.I. Munteanu, Yang Chunyan, V. Vladareanu, R.A. Munteanu, Li Weihua, F. Smarandache, A.I.Gal, Method and device for extension hybrid force-position control of the robotic and mechatronics systems, Patent, pp.20, OSIM A2012 1077/28.12.2012 